\title{
Spatiotemporal expression of matrix metalloproteinase-1 in progression of nonalcoholic steatohepatitis
}

\author{
Hiroaki Yokomori1,*, Yutaka Inagaki², Wataru Ando ${ }^{3}$, Mitsuko Hara $^{4}$, Takako Komiyama ${ }^{3}$, Soichi Kojima ${ }^{4}$, Masaya Oda ${ }^{5}$, Hajime Kuroda ${ }^{6}$, \\ Yutaka Suzuki ${ }^{7}$ and Isao Okazaki ${ }^{8,9}$ \\ 1 Department of General Internal Medicine, Kitasato University Medical Center, Saitama 364-8501, Japan \\ ${ }^{2}$ Center for Matrix Biology and Medicine, Tokai University Graduate School of Medicine, Kanagawa 259-1193, Japan \\ ${ }^{3}$ Department of Clinical Pharmacy, School of Pharmacy, Kitasato University, Tokyo, Japan \\ ${ }^{4}$ Micro-Signaling Regulation Technology Unit, RIKEN CLST, Saitama 351-0198, Japan \\ ${ }^{5}$ Department of Internal Medicine, Sanno Medical Center, International University of Health and Welfare, Tokyo 107-0052, Japan \\ ${ }^{6}$ Department of Pathology, Sanno Medical Center, International University of Health and Welfare, Tokyo 107-0052, Japan \\ ${ }^{7}$ Department of Surgery, Sanno Medical Center, International University of Health and Welfare, Tokyo 107-0052, Japan \\ ${ }^{8}$ Department of Internal Medicine, International University of Health and Welfare Hospital, Tochigi 329-2763, Japan \\ ${ }^{9}$ Department of Internal Medicine, Sanno Hospital, International University of Health and Welfare, Tokyo 107-0052, Japan
}

\begin{abstract}
Human matrix metalloproteinase-1 (MMP-1) has been shown to contribute to the regression of experimental liver fibrosis when ectopically expressed in rodents. It remains unknown whether the same mechanism exists in chronic liver diseases in humans. This study was conducted to elucidate the functional contribution of MMP-1 to the pathophysiology of nonalcoholic steatohepatitis (NASH). Samples from 19 histologically proven NASH patients were examined together with normal liver specimens from four subjects. Expression of MMP-1, caveolin-1, active and latent forms of TGF- $\beta$, and type 1 procollagen was analyzed using immunohistochemical, western blotting and immunoelectron microscopic (IEM) examinations. In the early stage of NASH, MMP-1 was expressed predominantly in Kupffer cells and monocytes. Remarkable expression of MMP-1 and caveolin-1 was observed in hepatic progenitor cells (HPCs), hepatic stellate cells (HSC) and proliferating capillary endothelial cells in the advanced stage of NASH. MMP-1 immunostaining was significantly greater in advanced $(p<0.014)$ and early $(p<0.016)$ NASH than in control. IEM revealed MMP-1-immunoreactive products in HPCs/HSC/small hepatocytes. Active form of TGF- $\beta$ and type I procollagen were detected at the edge of those capillary endothelial cells adjacent to the HPCs. MMP-1 expressed in HPCs/small hepatocytes/ capillary endothelial cells may contribute, at least in part, to the repair and regeneration of fibrotic liver through the caveolin-1 signal in the advanced stage of NASH.
\end{abstract}

Keywords: MMP-1; TGF- $\beta$; caveolin-1; nonalcoholic steatohepatitis; hepatic progenitor cells; spatiotemporal expression

\section{Introduction}

Nonalcoholic fatty liver disease (NAFLD) and nonalcoholic steatohepatitis (NASH) are growing in importance in both economically developed and developing countries due to lifestyles that include overeating and inadequate physical activity [1, 2]. Among NASH patients, 9\%-20\% develop cirrhosis with or without hepatocellular carcinoma (HCC) over a long period of time [3-6]. Many reports on NAFLD and NASH have discussed their epidemiology [3], morphological definition and classification [4], pathophysiology [1-6], innate immunity [7], natural clinical courses [3-6], and proposed diverse treatment strategies [1-5]. However, the underlying mechanisms responsible for the progression of fibrosis in NASH remain unclear.

Inflammasomes formed by the interaction of bacterial products with toll-like receptors (TLRs) cause hepatocyte injury, whichinturnengenderstheactivationofmacrophages and hepatic stellate cells (HSCS) and contributes to the progression of fibrosis in NASH $[8,9]$. Macrophages activated by either hepatocellular or biliary injury engender bi-potent progenitor proliferation depending on the type of injury, and cause both fibrosis and regeneration of the liver $[10,11]$. Furthermore, transforming growth factor- $\beta$ (TGF- $\beta$ ) signaling in hepatocytes has also been implicated in promoting steatosis and fibrosis in NASH [12].

*Corresponding author: Hiroaki Yokomori, Department of Internal Medicine, Kitasato University Medical Center, Saitama 364-8501, Japan. Tel.: +81-48-593-1212; Fax: +81-48-593-1239; E-mail: yokomori@insti. kitasato-u.ac.jp

Received 13 January 2016 Revised 12 March 2016 Accepted 21 March 2016 Published 30 March 2016

Citation: Yokomori H, Inagaki Y, Ando W, Hara M, Komiyama T, Kojima S, Oda M, Kuroda H, Suzuki Y, Okazaki I. Spatiotemporal expression of matrix metalloproteinase-1 in progression of nonalcoholic steatohepatitis. J Mod Hum Pathol. 2016; 1(2):11-20. DOI:10.14312/2397-6845.2016-3

Copyright: (c) 2016 Yokomori H, et al. Published by NobleResearch Publishers. This is an open-access article distributed under the terms of the Creative Commons Attribution License, which permits unrestricted use, distribution and reproduction in any medium, provided the original author and source are credited. 
Matrix metalloproteinase-1 (MMP-1), the major interstitial collagenase in humans, can degrade collagen molecules at the triple helical domain and regulate extracellular matrix turnover in tissue [13]. A previous report has shown that forced expression of human MMP-1 in rat fibrotic liver suppresses fibrogenesis and contributes to recovery from experimental liver fibrosis [14]. In addition, MMP-1 is known to induce human capillary tubular network collapse, leading to regression [15] as well as to activate TGF- $\beta$ [16], the key molecule that accelerates tissue fibrosis. Little is known, however, about the endogenous expression and pathophysiological role of MMP-1 in human liver diseases including NAFLD and NASH.

The present study was conducted using liver biopsy specimens obtained from NASH patients to examine the localization of MMP-1, caveolin-1, active (aTGF- $\beta$ ) and latent (ITGF- $\beta$ ) forms of TGF- $\beta$, as well as the markers of hepatic progenitor cells (HPCs) such as OV-6 and CK-19. The authors aim to elucidate the functional contribution of MMP-1 to the pathophysiology of early and advanced stages of NASH, especially in relation to the fibrogenic and regeneration processes. The results indicate that MMP-1, which was expressed in different types of cells in the early and advanced stages of NASH, contributes consistently to disease progression. Especially in the advanced stage of NASH, MMP-1 expressed in HPCs/small hepatocytes/ capillary endothelial cells may contribute, at least in part, not only to the repair and regeneration of fibrotic liver through the caveolin-1 signal, but also to the regression of capillary endothelial cells to form angiogenesis that leads to liver cirrhosis.

\section{Methods}

Patients: This study examined 19 histologically proven NASH patients and 4 normal liver samples (Supplementary Table 1). The normal liver samples were collected from 4 subjects who underwent hepatic resection because of metastases of colon cancer, and all normal samples were collected from at least $30 \mathrm{~mm}$ or more distant from the tumor. Liver specimens from 17 of 19 patients were examined together with 4 normal liver samples for the Computer-assisted Morphometric Analysis. Eight of 17 patients were examined histological co-expression analysis. Eight of 19 patients and 3 of 4 normal samples were examined western bolt analysis. This study was approved by the relevant ethics committees of the institutions involved. Informed consent was obtained from all subjects. The clinical diagnostic criteria used are described under supplementary materials and methods (Appendix-I).

Histological examination: Conventional histological examination was performed using hematoxylin and eosin, Azan-Mallory, and silver staining. Assessment of the histological stages of NASH was conducted using Matteoni classification [4], Brunt's classification [17] and the NASH activity score (NAS) [18]. Details related to dual immunohistochemical (IHC) and immunoelectron microscopic (IEM) examinations, western blot analysis and bioassays for serum cytokines are presented in supplementary materials and methods (Appendix-I).

Antibodies: Specific antibodies were purchased and used to detect MMP-1 (Abcam plc., Cambridge, UK), caveolin-1
(NB 100-615; Novus Biologicals, Littleton, CO, USA), ITGF- $\beta$ (Cosmo Bio Co. Ltd., Tokyo, Japan), CD34 (clone QBEnd 10; Dako, Glostrum, Denmark), CD68 (clone PG-M1; Dako), vimentin (clone V9; Dako), type I procollagen (Santa Cruz Biotechnology Inc., Santa Cruz, CA, USA), OV-6 (R\&D Systems, Minneapolis, MN, USA), and cytokeratin 19 (CK19, clone RCK108; Dako). Antibodies recognizing aTGF- $\beta$ were prepared by Hara et al. as reported previously [19].

Immunohistochemical expression of MMP-1, caveolin-1, aTGF- $\beta$, ITGF- $\beta$, type I procollagen

Immunohistochemical analysis was conducted as reported earlier [20], with minor modifications. Serial sections of formalin-fixed, paraffin-embedded tissue sections $(4 \mu \mathrm{m})$ were deparaffinized and rehydrated by immersion in xylene and graded alcohol concentrations. Endogenous peroxidase activity was quenched by incubation in $0.3 \%$ $(\mathrm{v} / \mathrm{v}) \mathrm{H}_{2} \mathrm{O}_{2}$ in methanol for 30 min at room temperature (RT). Heat-mediated antigen retrieval was used for autoclave treatment ( $10 \mathrm{mM}$ citrate buffer; $\mathrm{pH} 6.0$ ) for $15 \mathrm{~min}$ at $120^{\circ} \mathrm{C}$. Sections were blocked with $10 \%$ normal goat serum (Vector Laboratories Inc., Burlingame, CA, USA) and incubated for 90-120 min with primary antibody in $2 \%$ serum. They were incubated overnight at $4^{\circ} \mathrm{C}$ with $1: 100$ dilution of anti-MMP-1, anti-caveolin-1, anti-activated form of TGF- $\beta$ (a TGF- $\beta$ ), antilatent form of TGF- $\beta$ (ITGF- $\beta$ ), or anti-type 1 procollagen. The sections were incubated with the $\mathrm{N}$-Histofine ${ }^{\circledR}$ Simple Stain MAX PO (Nichirei Corp., Tokyo, Japan) at RT for 30 min. After repeated washing with PBS, the sections were reacted with diaminobenzidine containing $0.01 \% \quad \mathrm{H}_{2} \mathrm{O}_{2}$ and counterstained with hematoxylin for light microscopic study. Immunoelectron microscopy was performed using immunogold-silver enhancement of control and NASH liver samples according to previous reports. Details of the methods described above are presented in supplementary materials and methods (Appendix-I).

Computer-assisted morphometric analysis and western blot analysis are also presented in supplementary materials and methods (Appendix-I).

\section{Statistical analysis}

Values are expressed as mean \pm SD. Mann-Whitney $U$ tests were used to evaluate differences between groups: a $p$ value $<0.05$ was regarded as statistically significant.

\section{Results}

\section{Histological findings and stage classification}

According to Matteoni classification [4], 8 cases were diagnosed as type 4. All others were type 3. Using Brunt's grading [16], 7 cases were categorized as grade 1, 5 cases as grade 2, and the others as grade 3 (Supplementary Figure 1). The histological findings obtained with Brunt's staging and NAS scoring methods [17] for all enrolled patients are presented in supplementary table 1 (Appendix-II). According to the results, patients were classified into the two groups shown in supplementary table 1 (AppendixII). Group 1 (early stage of NASH) comprised 10 cases of steatosis with mild inflammation, slight ballooning and slight-to-moderate fibrosis. Group 2 (advanced stage of NASH) included 9 cases of steatosis with marked inflammation, ballooning, and severe fibrosis or cirrhosis with or without HCC. 
MMP-1 expression in normal liver and early stage of NASH In normal liver, MMP-1 expression was observed in a small number of hepatocytes, LSECs, and blood vessels with very weak immunoreactivity (Figure 1a). In contrast, immunoreactive MMP-1 was localized in inflammatory cells present in the portal tracts and pericentral areas in the early stage of NASH (Figure 1b). Double staining using specific cell marker antibodies revealed that most of the MMP-1-expressing cells were CD68-positive Kupffer cells (KCs) and monocytes (Figure 1c). The others were vimentin-positive cells, possibly HSCs (data not shown). The MMP-1 expression was seldom co-localized with CD34 (data not shown). In fact, IEM observation revealed that gold-labeled MMP-1 particles were detected mostly within the cytoplasm of KCs and monocytes (Figures $1 \mathrm{~d}$ and 1e). Moreover, HSCs and LSECS showed extremely weak MMP-1 immunoreactivity in the early stage of NASH (data not shown).
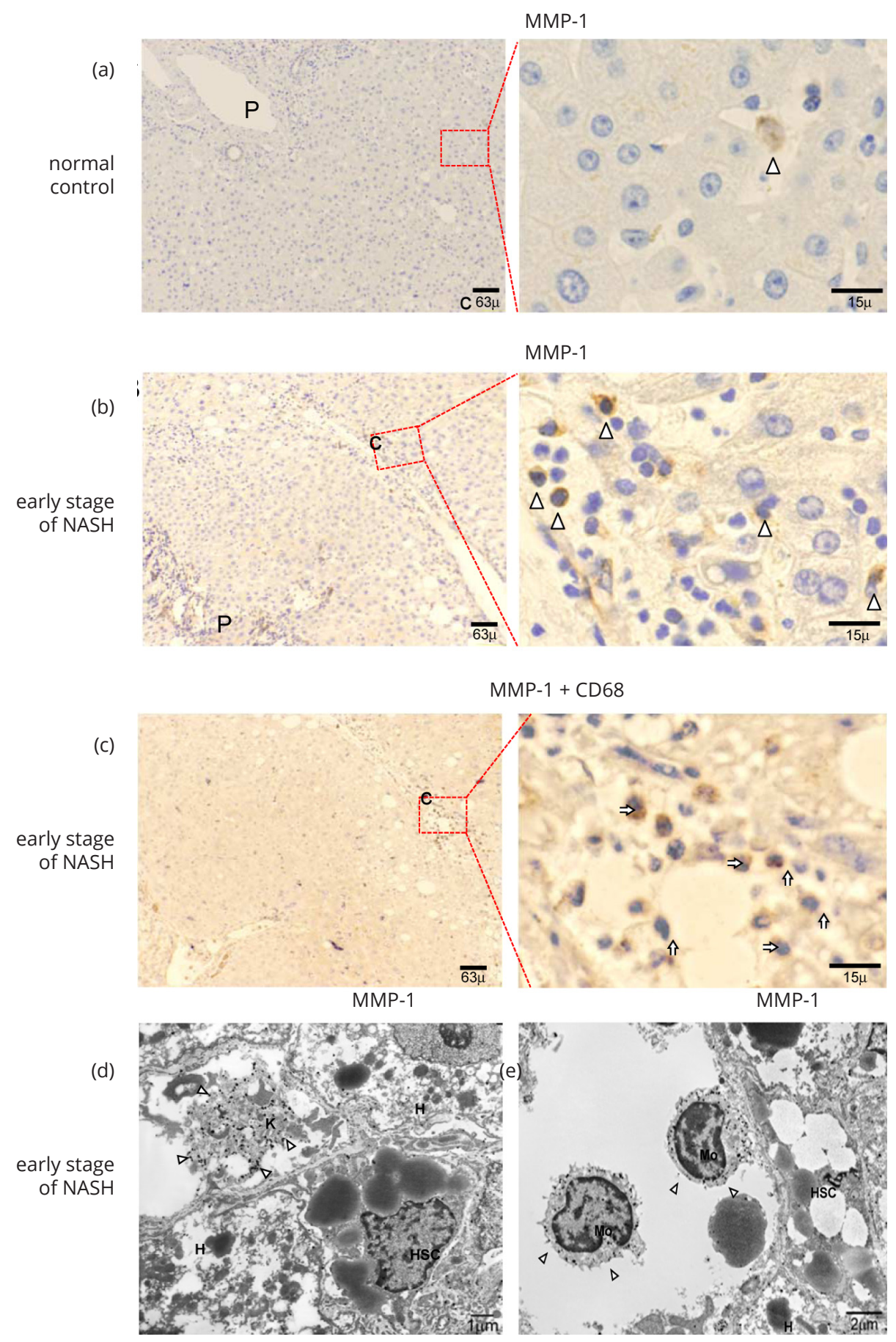

Figure $1 \mathrm{IHC}$ and IEM expression of MMP-1 in early stage NASH: (a) IHC expression of MMP-1 (brown) in normal liver (Case No. 1). A few cells (HCS, KCs, blood vessels) showed slight expression of MMP-1 in control liver. 100× magnification. The inset shows high magnification (400 $\times$ ); (b) IHC expression of MMP-1 (brown) in early stage NASH (Case No. 10). Definite expression of MMP-1 was observed in inflammatory cells in portal tracts and around pericentral areas in an early stage of NASH. 100× magnification. The inset shows high magnification (400×); (c) Dual IHC expression of MMP-1 (brown) and CD68 (blue) in early stage NASH (Case No. 10). Most MMP-1-positive cells were CD-68-positive KCs and monocytes. 100× magnification. Column denotes high magnification (400 $\times$ ): P, portal tract; C, central vein. Arrows indicate KCs or monocytes; (d, e) IEM expression of MMP-1 in early stage NASH (Case No. 8). Immunogold particles of MMP-1 are readily apparent in KCs and monocytes in early NASH liver. Arrowheads indicate MMP-1 reaction products. Scale bar shows magnification: H, hepatocyte; HSC, hepatic stellate cell; K, Kupffer cell; Mo, monocyte. 


\section{Caveolin-1 expression in normal liver and early stage of} $\mathrm{NASH}$

IHC analysis identified caveolin-1 on the walls of the hepatic artery, capillary venule, and portal vein in the portal tracts of normal liver tissues. Caveolin-1 expression was also detected in hepatic sinusoidal lining cells in pericentral zone 3, but only slightly in zones 1-2 of control normal liver (Supplementary Figure 2a). Caveolin-1 expression showed a similar pattern in the early stage of NASH (Supplementary Figure 2b). IEM observation revealed gold-labeled particles detecting caveolin-1 in non-coated vesicles or vacuoles in LSECs present in the same area (Supplementary Figure 2c). Similar findings were observed in the early stage of NASH (data not shown). No positive finding was obtained with regard to TGF- $\beta$ or type I procollagen expression in normal liver or early stage of NASH (data not shown).

\section{MMP-1 expression in advanced stage of NASH}

In the advanced stage of NASH, cells positive for MMP-1 were localized in the margins between the parenchyma and portal tracts, where ductular proliferation was observed (arrowheads in Figure 2a and Supplementary
Figure 3a). MMP-1 expression was also evident on the walls of capillarized endothelial cells in the peripheral region of fibrous septa (long arrows in Figure 2a and Supplementary Figure 3a). IHC analyses indicated that most MMP-1expressing cells present in the margin of portal tracts were OV-6-positive (Figure 2b and Supplementary Figure 3b) and/ or CK-19-positive (Figure 2c and Supplementary Figure 3c) hepatic progenitor cells (HPCS). In marked contrast to the findings obtained for the early stage of $\mathrm{NASH}$, expression levels of MMP-1 were rather low in KCs and monocytes (Supplementary Figure 3d). It is of particular interest that MMP-1-expressing HPCs stained positive for OV-6 (Figure 2b) and/or CK-19 (Figure 2c) aggregated to form a cell cluster structure. To further confirm the expression of MMP-1, we conducted an IEM examination. Gold-labeled MMP-1 particles were observed on the luminal surfaces and intracellular organelles of HPCs (Figure 2d) and small hepatocytes (Figure 2e). Taken together, the extent of MMP-1 expression in HPCs and small hepatocytes increased parallel with the degree of progenitor cell reaction, suggesting that MMP-1 may participate in the repair and regeneration of hepatocytes.
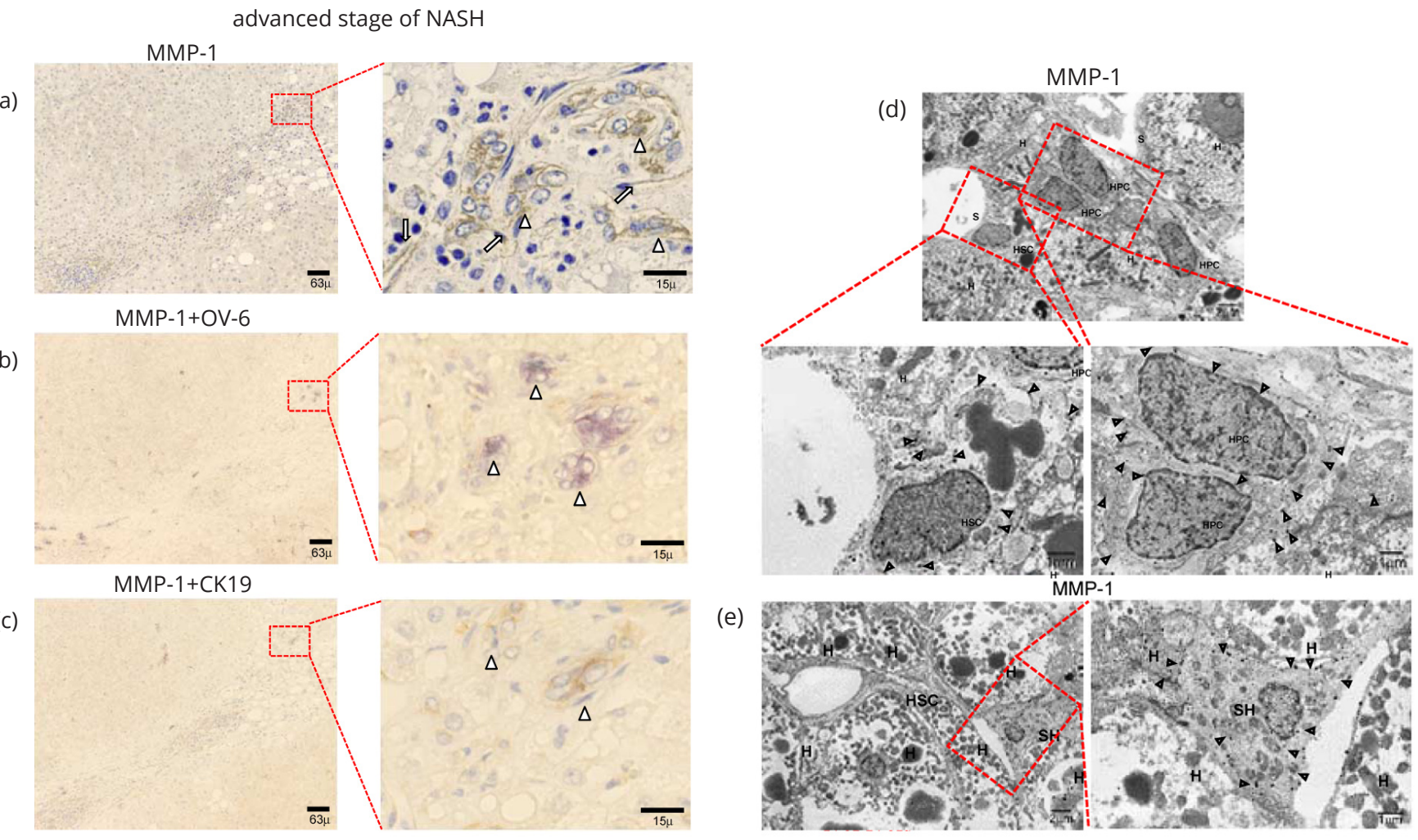

Figure 2 IHC and IEM expression of MMP-1 in advanced stage NASH using serial sections (Case No. 17): (a) Expression of MMP-1 is localized on sinusoidal lining cells in steatosis with features of injured cells and inflammation. Expressions of MMP-1 increased concomitantly with the development of fibrosis. The long white arrow indicates the MMP-1-positive capillarized endothelial cells. White short arrows indicate MMP-1-positive cells within fibrous septa presumed to be HPCs; (b) IHC dual expression of MMP-1 (brown) and OV-6 (blue). Cells showing dual expression are apparently HPCs (small arrows). 100x magnification. Column denotes high magnification (400x); (c) IHC dual expression of MMP-1 (brown) and CK-9 (blue). Dual positive cells differed from cells positive for MMP-1 and OV-6. Cells showing dual expression are apparently HPCs (small arrows). 100x magnification. Column denotes high magnification (400×); (d) Immunogold-labeled MMP-1 particles are observed on HPCs, distributed on luminal surfaces and intracellular organelles of HPCs. Upper panel denotes low magnification. Lower two panels denote high magnification; (e) Immunogold-labeled MMP-1 particles are observed on the plasma membrane of small hepatocytes, distributed on luminal surfaces and intracellular organelles of hepatic cells. Scale bars in each Figure show magnification. Arrowheads indicate MMP-1 reaction products: $\mathrm{E}$, endothelial cells; $\mathrm{H}$, hepatocytes; HPC, hepatic progenitor cells; HSC, hepatic stellate cells; $\mathrm{S}$, lumen of sinusoid; SH, small hepatocyte.

\section{Caveolin-1 expression in advanced stage of $\mathrm{NASH}$}

IHC examination revealed caveolin-1 expression in the advanced stage of NASH in hepatic sinusoidal lining cells in periportral zone 1 (long arrows in Figure $3 a$ and
Supplementary Figure 3e). Positive staining was also detected in the walls of HPCs (arrowheads in Figure 3a and Supplementary Figure 3e). IEM studies revealed goldlabeled particles of caveolin-1 on caveolae-like structures 
of capillarized endothelial cells and HPCs in the advanced stage of NASH (Figure 3b).

\section{Role of MMP-1 and caveolin-1 in fibrogenesis in NASH}

Dual IHC staining revealed a cell cluster structure of MMP1-expressing cells that was lined by vimentin-positive mesenchymal cells (Figure $3 c$ and Supplementary Figure 4a). Moreover, cells expressing type I procollagen (Figure
$3 d$ and Supplementary Figure $4 b$ ) and/or aTGF- $\beta$ (Figure $4 a$ and Supplementary Figure 4c) were observed at the edge of a cell cluster structure, indicating active fibrogenesis in those areas. In contrast, ITGF- $\beta$ expression was detected in the fibrous septa (Figure $4 \mathrm{~b}$ and Supplementary Figure 4d), but not around the cell cluster structures. IEM observations also revealed type 1 procollagen-producing cells with elongated fiber-like projections at the edge of the cell cluster structure composed of HPCs (Figure 4c).

Advanced stage of NASH

(a)

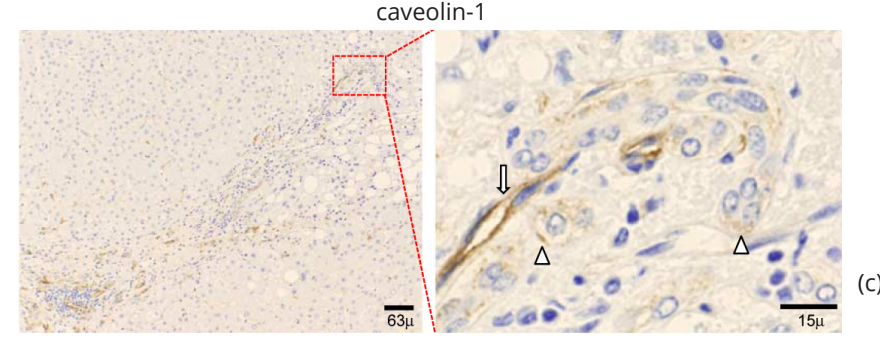

caveolin-1

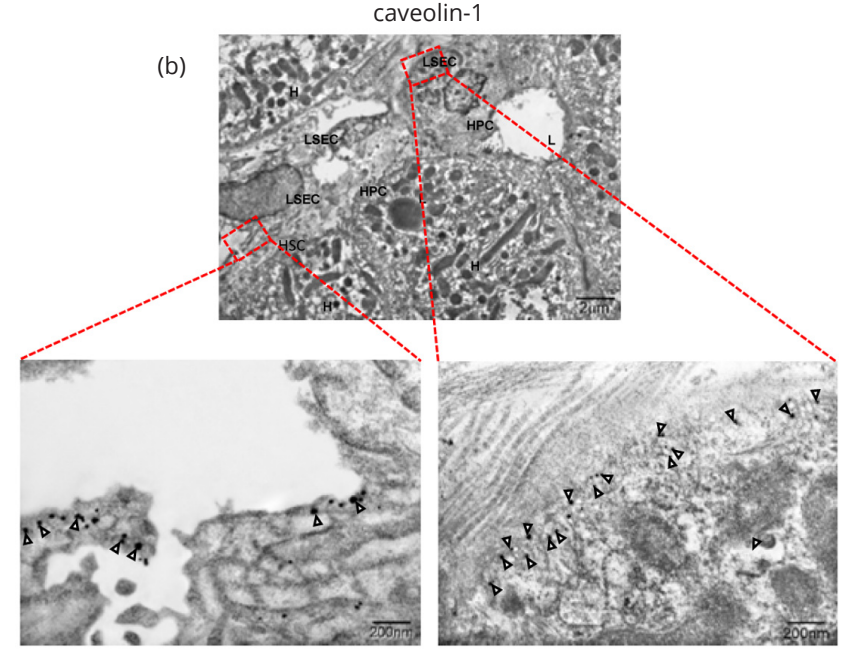

(c)

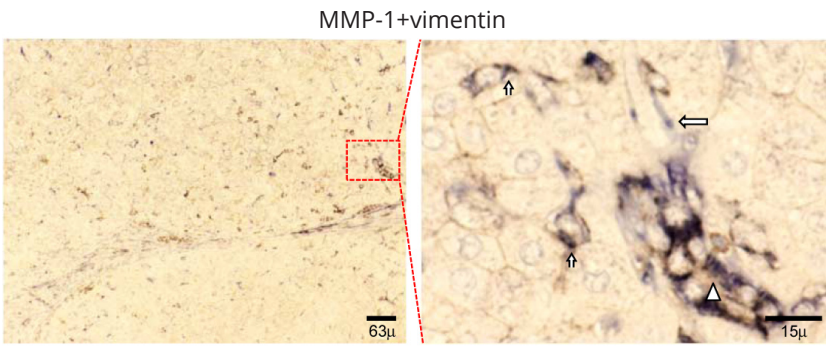

(d)

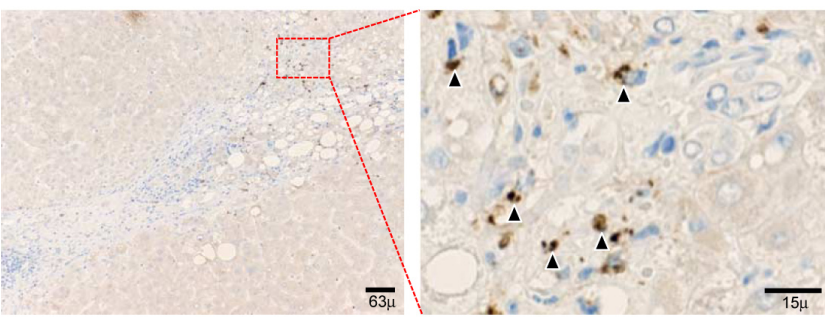

Figure 3 IHC and IEM expression of caveolin-1, MMP-1/ vimentine, and type 1 procollagen in advanced stage NASH using serial sections (Case No. 17): (a) IHC expression of caveolin-1 in advanced stage NASH. The high magnification panel shows caveolin-1 expression in hepatic sinusoidal lining cells in the periportal zone 1 (white long arrow). Short arrows indicate positive staining in the cell walls of HPCs. 100× magnification. Column denotes high magnification $(400 \times)$; (b) IEM expression of caveolin-1 in advanced stage NASH. Highly magnified image, circled with a red dotted line. Immunogold particles of caveolin-1 are observed on caveolae-like structures of HPCs (right) and capillarized endothelial lining cells (left). The scale bar shows the magnification in each figure. Arrowheads indicate caveolin-1 reaction products: $\mathrm{H}$, hepatocytes; HPC, hepatic progenitor cells; HSC, hepatic stellate cells; L, lumen of sinusoid; LSEC, liver sinusoidal endothelial cells; SH, small hepatocyte; (c) Dual expression of MMP-1 (brown) and vimentin (blue). IHC dual staining shows a cell cluster structure observed clearly in the region of ductular reaction; (d) Type 1 procollagen expression (brown). The cell cluster structures connect the newly formed capillarized endothelial lining cells; type 1 procollagen-producing cells are observed at the edges.

\section{Computer-assisted morphometric analysis and western blotting}

We assessed morphometric variables determined by labeling MMP-1 in three portal regions and regenerative fibrotic areas. In the periportal tract, MMP-1 immunostaining was 0.342 pixels $/ \mathrm{mm}^{2}$ in control, 0.766 pixels $/ \mathrm{mm}^{3}$ in early $\mathrm{NASH}$, and 1.513 pixels $/ \mathrm{mm}^{2}$ in advanced $\mathrm{NASH}$. It was significantly greater in advanced $(p<0.014)$ and early $(p<0.016) \mathrm{NASH}$ than in control (Figure $4 d)$.

To confirm the immunohistochemical results and morphometric analysis for MMP-1 expression, we investigated the protein expression of MMP-1 in control liver, early stage of $\mathrm{NASH}$, and advanced stage of NASH samples using western blotting. MMP-1 expression in control samples was scant. However, MMP-1 expression was elevated in early NASH and even higher in advanced
NASH samples. Quantitative densitometric data (expressed as MMP-1/actin levels for each group) from multiple samples are shown as mean \pm SEM. MMP-1 protein was expressed abundantly in early NASH and even more in advanced NASH (Figure 4e).

Next, we estimated the co-expression of MMP-1 in various cell marker-positive cells using digital image processing. Data for the periportal tract on MMP-1 expression of each cell-marker immunostaining expression in both early and advanced NASH are shown in figure 5. MMP-1 expression by CD68 and vimentin pixels were significantly higher than that by OV-6 and CK-19 pixels in early NASH, while MMP-1 expression by OV-6 and CK19 pixels were significantly higher than that by CD68 pixels in advanced NASH (Table 1 and Figure 5). 


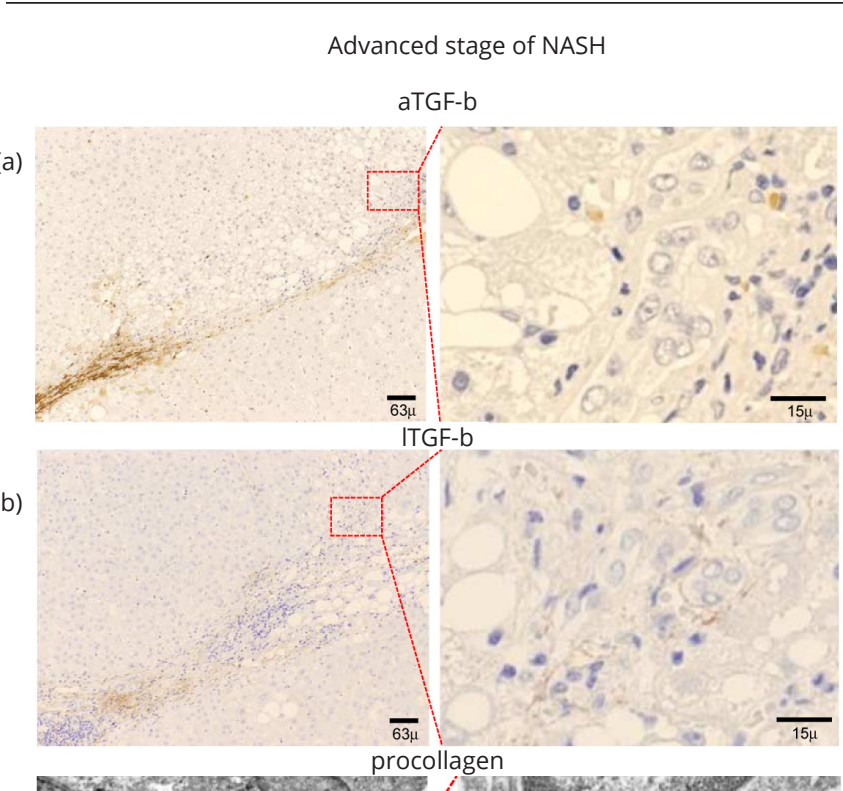

(c)
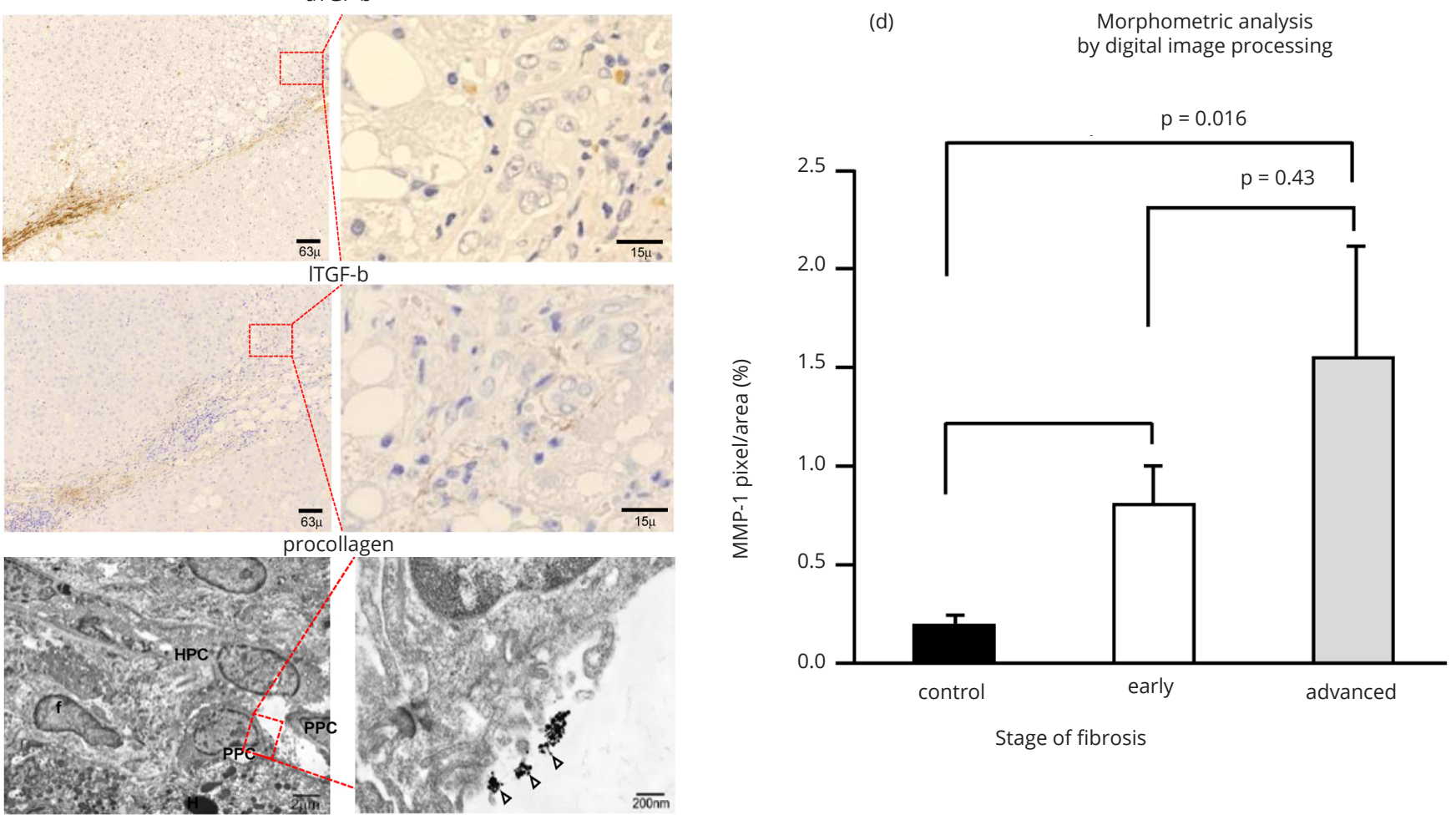

(e) Western blotting

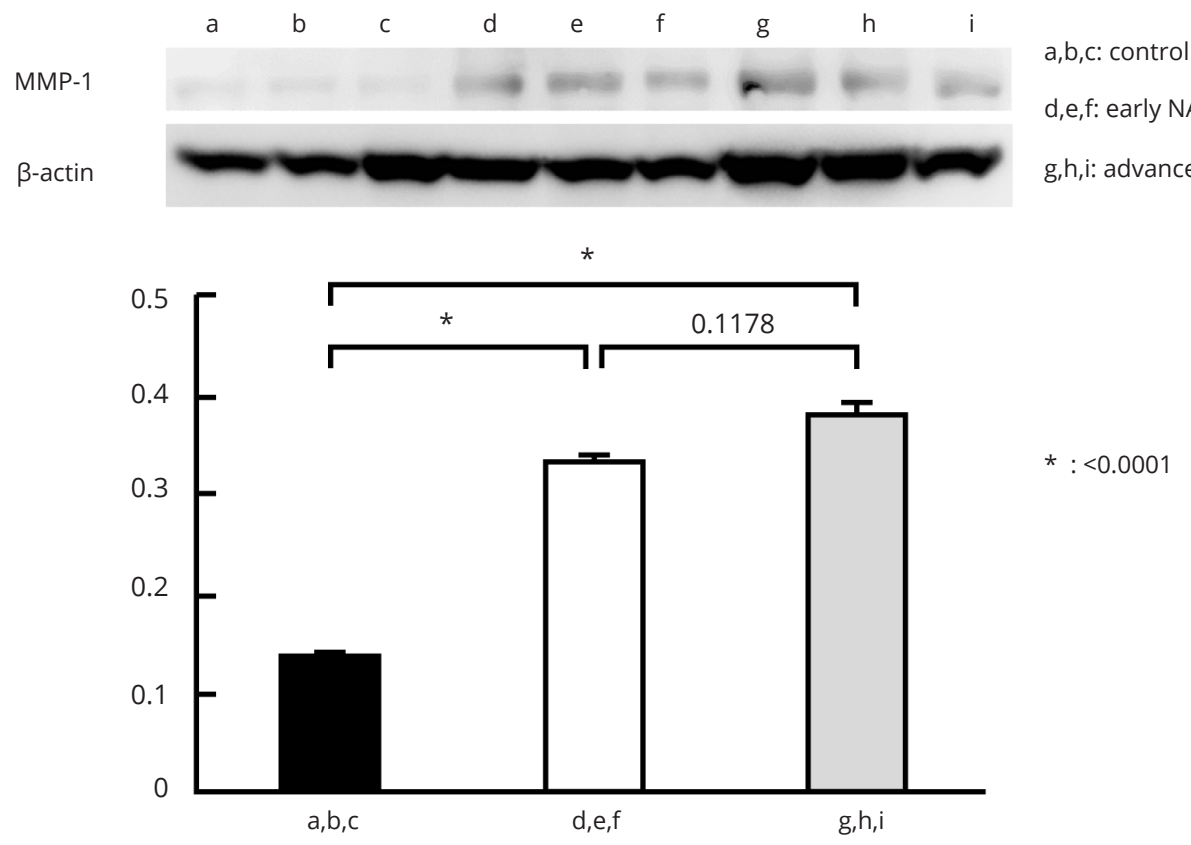

Figure 4 IHC expression of aTGF- $\beta$ and ITGF- $\beta$ in advanced NASH using serial sections, computer-assisted morphometric analysis and western blotting: (a) aTGF- $\beta$ expression (brown). Increased expression of aTGF- $\beta$ is observed in cells neighboring a cell cluster structure, but positive cells do not express type I procollagen (Case No. 15); (b) ITGF- $\beta$ expression (brown). ITGF- $\beta$ expression is detectable in the fibrous septa of NASH with fibrosis. $100 \times$ magnification. Column denotes high magnification $(400 \times)$. White arrowheads indicate hepatic progenitor cells. Black arrowheads indicate vimentin-positive cells. White arrowheads indicate proliferative capillary arteries or sinusoidal lining cells (Case No. 15); (c) IEM expression of type 1 procollagen in advanced NASH. Upper panel denotes lower magnification. Lower panel denotes higher magnification. Type 1 procollagen-reactive particles are observed on elongated fiber-like projections of procollagen-producing cells adjacent to HPCs denoted by red circles. Scale bar shows the magnification in each figure. Arrowheads indicate procollagen reaction products: $\mathrm{H}$, hepatocytes; HPC, hepatic progenitor cells; PPC, type 1 procollagen-producing cells (Case No. 14); (d) Morphometric analyses of MMP-1 immunostaining in control and NASH liver samples. MMP-1 are expressed in pixels per square millimeter. Percentage was calculated by the mean of different 3 sections in each patients and normal samples. Data are mean \pm SEM. Statistical analyses were conducted using one-way ANOVA followed by Dunn post-hoc analysis; (e) Western blot analysis of MMP-1 protein in human control and NASH liver tissues. Samples containing $30 \mu g$ protein were subjected to SDS-PAGE and analyzed by western blotting. Lanes a-c show normal control liver samples (Case No. 1, 2, 3). Lanes d-f show early NASH liver samples (Case No. 6, 8, 9, 10). Lanes g-i show advanced NASH liver samples (Case No. 12, 17, 18, 19). MMP-1 protein expression increased significantly in early NASH and even higher in advanced NASH liver. 


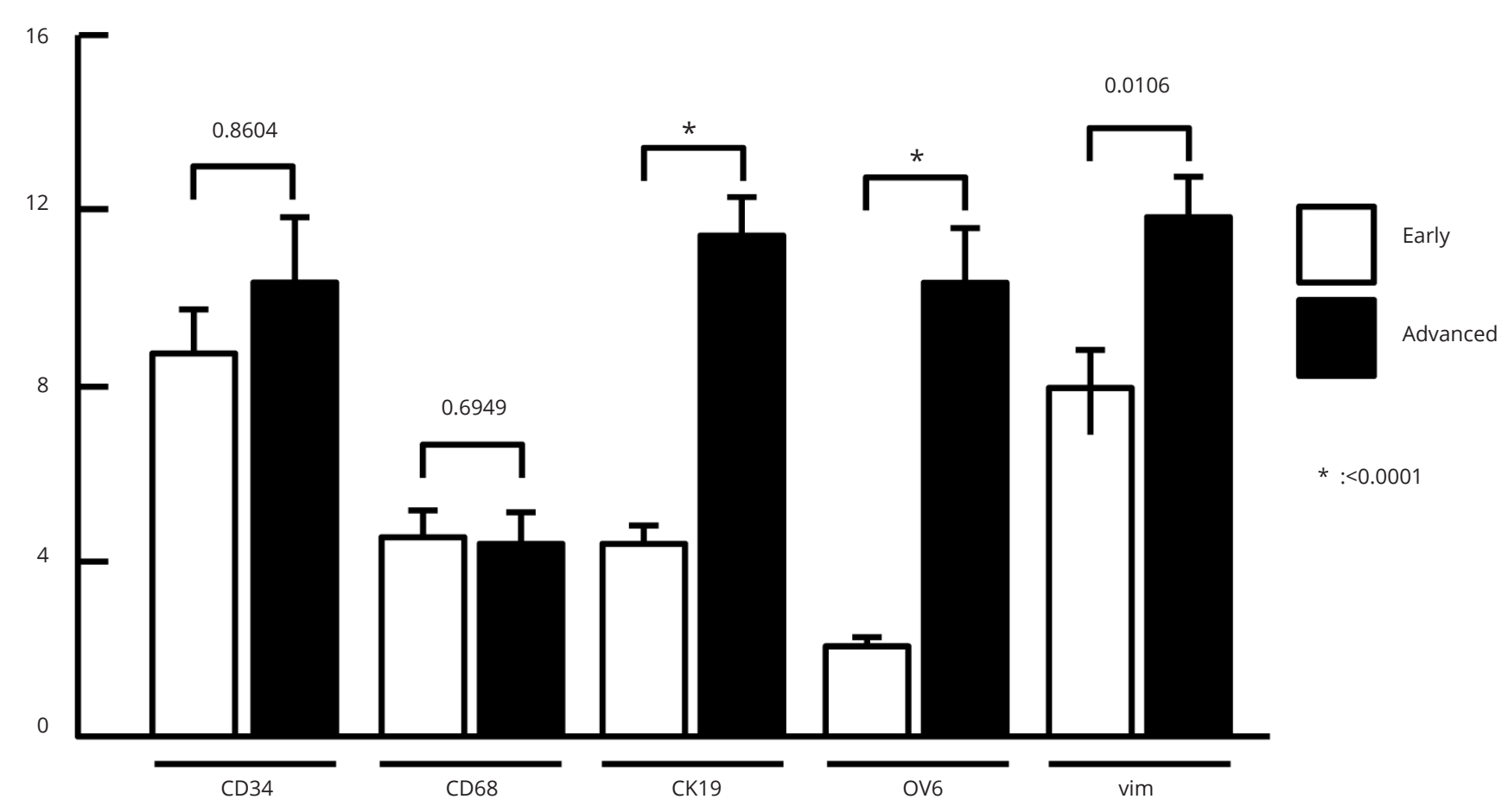

Figure 5 Computer-assisted morphometric analysis of co-expression of MMP-1 and cell markers using digital image processing. The percentage of pixels was the mean of co-expression staining pixels in the photographs. Photographs were examined for each 4 patients by different 5 sections in each antibody. (a) Comparison of MMP-1 expression pixels by various cell marker expression pixels shown by percentage; (b) Pixel data of MMP-1 expression in early NASH shown by each type of cell marker compared with those in advanced NASH indicated as percentage.

Table 1 The co-expression of MMP-1 in each cell-markers-positive cells.

\begin{tabular}{cccc}
\hline Cell markers & $\begin{array}{c}\text { Early stage of } \\
\text { NASH }\end{array}$ & $\begin{array}{c}\text { Advanced stage } \\
\text { of NASH }\end{array}$ & $\begin{array}{c}\text { Increase or } \\
\text { decrease rate }\end{array}$ \\
\hline CD34 & $8.73 \pm 0.99 *$ & $10.33 \pm 1.49$ & $-18.3 \%$ \\
CD68 & $4.55 \pm 0.61$ & $4.43 \pm 0.69$ & $-2.6 \%$ \\
CK-19 & $4.39 \pm 0.43$ & $11.42 \pm 0.95$ & $+160 \%$ \\
OV-6 & $2.04 \pm 0.24$ & $10.33 \pm 1.27$ & $+406 \%$ \\
Vimentin & $7.90 \pm 0.91$ & $11.84 \pm 0.86$ & $+49.9 \%$ \\
\hline
\end{tabular}

*Unit; MMP-1 expression pixels/cell marker expression pixels.

\section{Roles of MMP-1 and caveolin-1 in angiogenesis in NASH}

As noted above, both caveolin-1 and MMP-1 were expressed in caveolae like structures of capillarized endothelial cells and HPCs. The capillarized endothelial lining cells were connected to the edge of a cell cluster structure. IHC dual staining of MMP-1 and CD 34 suggested that the capillarized endothelial lining cells were both positive. They induced repair and regeneration of the injured sinusoidal structure (Supplementary Figure 4e).

In caveolae of hepatic stellate cells, IEM examination revealed immunogold particles showing MMP-1 (Figure $3 d)$ as well as in the caveolae-like structures of capillarized sinusoidal endothelial cells (Figures $6 a$ and $6 \mathrm{~b}$ ). The angiogenic sprouting of capillary endothelial cells was flat and elongated (Figure 6b), and MMP-1 particles were expressed in caveolae along the luminal and abluminal portions of the cell membrane (Figure $6 b$ ). Taken together, type 1 procollagen-producing cells (arrowheads in Figures $3 d$ and $4 c$ ) were linked to capillary endothelial lining cells with expression of both MMP-1 and caveolin-1.

\section{Discussion}

In this study, we have shown for the first time increased expression and distinct localization of MMP-1 during the progression of NASH in humans. It is expressed predominantly in monocytes and KCs in the early stage of NASH, while it is detected in HPCs and proliferating capillary endothelial cells in the advanced stage of the disease. Because of the biological function of MMP-1 in degrading extracellular matrix (ECM) components, prior to conducting this study we expected the expression levels of MMP-1 to be lower in the advanced stage of NASH with fibrosis or cirrhosis than in the early stage of NASH without discriminate fibrosis. The findings of the present study suggest that the functional role of MMP-1 differs greatly from reports to date on MMP-1 as the key enzyme of ECM metabolism.

Regarding the functional role of MMP-1, recombinant adenoviral delivery for MMP-1 activation in endothelial cells resulted in capillary tube regression of human umbilical endothelial cells in an in vivo study [15]. Our findings for the advanced stage of NASH are consistent with this in vivo study, suggesting the possibility of functions of MMP-1 beyond that as the key enzyme of matrix degradation. This is discussed below.

Another important purpose of this study is to clarify the role of caveolin-1 on MMP-1 expression in the pathophysiology of NASH because up-regulation of caveolin-1 expression was reported in a murine NAFLD model [21]. The results of the present study also suggest co-localization of MMP-1 and caveolin-1 in caveolae under pathological conditions. Although MMP-2 [22], MMP-923 and MT1-MMP [24] have 
Advanced stage of NASH
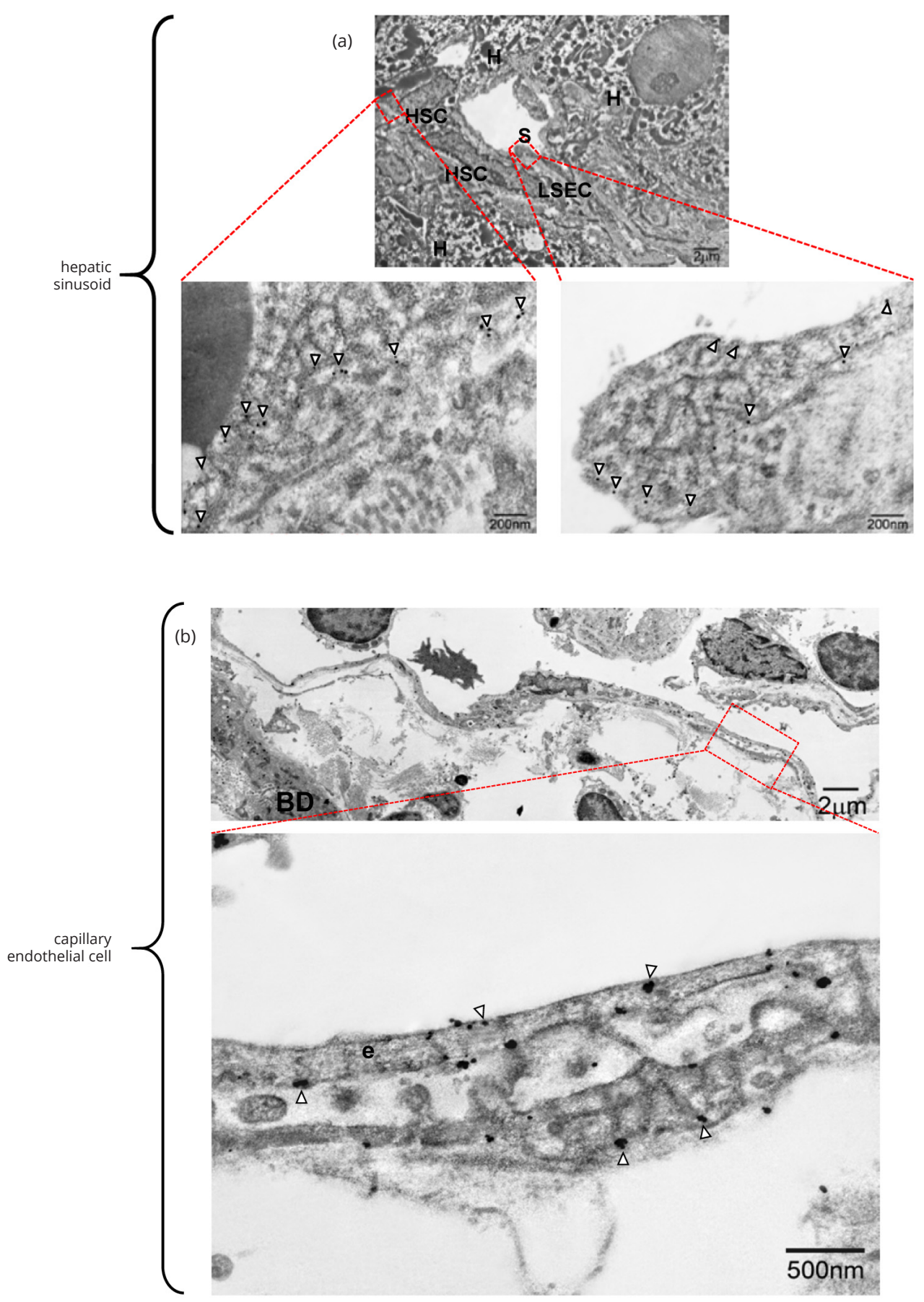

Figure 6 IEM expression of MMP-1 related to angiogenesis and fibrosis in advanced NASH: (a) Liver sinusoidal lining cells. MMP-1-reactive immunogold particles are expressed on caveolae of hepatic stellate cells (left lower panel) and caveolae-like structures of liver capillarized sinusoidal endothelial cells (right lower panel). The scale bar shows the magnification in each figure. Arrowheads indicate MMP-1 reaction products: SEC, sinusoidal endothelial cell; (b) Capillary endothelial cell (upper panel). Sprouting capillary endothelial cells are flat and elongate. Nucleic portions are slightly elevated. The lumen surface is irregular. Scale bar shows the magnification. BD, bile ductule. Highly magnified image (lower panel), circled with a red dotted line. MMP-1-positive particles are expressed on caveolae along the luminal and abluminal portions of the cell membrane of capillary endothelial cells. Scale bar shows magnification. Arrowheads indicate MMP-1 reaction products.

been reported to be co-localized with caveolin-1, MMP-1 has not been reported to be expressed in caveolae. CD147/ EMMPRIN is known to form a complex with caveolin-1 on the surface of cells of multiple types [25]. Glycosylation of CD147 up-regulates MMP-1 expression [26] whereas negative regulation of CD147 by caveolin-1 engenders decreased CD147-dependent MMP-1 induction [25, 26]. These findings, combined with the results of this study, suggest that MMP-1 expression is regulated through the caveolin-1 signal.

In the early stage of $\mathrm{NASH}$, steatosis and the consequent inflammation cause activation of $\mathrm{KCS}$ that secrete chemokine CCL2 (MCP-1) to recruit monocytes [27]. MMP-1 expression in those cells may be up-regulated by the interaction with caveolin-1, as described above, and MMP- 
1-positive monocytes appear to differentiate into activated macrophages. KCs, monocytes, and macrophages have been reported to stimulate differentiation of hepatic stem cells into HPCs [28], and accelerate ductular reaction composed of proliferating HPCs $[10,27]$.

In the advanced stage of NASH, IEM observations revealed that HPCs and small hepatocytes with increased MMP-1 expression may represent injured liver regeneration. The microenvironment around cells is known to be the most important factor regulating their differentiation through the caveolin-1 signal. An excess amount of lipids attenuates HPC differentiation [29].

Dual staining of the serial sections revealed that a cell cluster structure composed of MMP-1+ OV-6+ CK19HPCs extended toward the central zone within the lobules. They were connected to MMP-1+ capillary endothelial lining cells. These findings suggest that MMP-1+ HPC may differentiate into capillary endothelial cells as described in a previous report [15]. MMP-1 activation by serine proteases induced human capillary tubular network collapse and regression in 3D collagen matrices that was evidenced by siRNA targeting MMP-1 [15]. Moreover, several recent studies of angiocrine signals have demonstrated their stimulatory effects on liver regeneration after injury [3032]. One of those studies demonstrated that expression of CXCR7 and CXCR4 (a receptor for SDF-1 receptor) was up-regulated in LSECS and induced liver regeneration after acute injury, although constitutive FGFR1 signaling in LSECs inhibited CXCR4 expression after chronic liver injury followed by fibrosis [30]. Liver sinusoidal endothelial cells are responsible for nitric oxide modulation [33]. In an earlier study we observed increased expression of caveolin-1 in human cirrhotic liver in relation to nitric oxide [34]. Portal venous blood in NASH patients contains high levels of sugar, lipids, amino acids, and inflammasomes, and hepatic sinusoidal endothelial cells act as a gatekeeper to prevent hepatocyte injury. It is interesting and important to ascertain how the MMP-1/caveolin-1 signal in HPCs and endothelial cells works in pathophysiological conditions such as regeneration of fibrotic liver.

In further studies, the authors aim to clarify the novel function of MMP-1 beyond the destruction of extracellular matrix. Translational research targeting the MMP-1/ caveolin-1 signal should be conducted to improve treatment of NASH.

\section{Conclusion}

IEM revealed MMP-1-immunoreactive products in HPCS/ $\mathrm{HSC} / \mathrm{small}$ hepatocytes. Active form of TGF- $\beta$ and type I procollagen were detected at the edge of those capillary endothelial cells adjacent to the HPCs. MMP-1 expressed in HPCs/small hepatocytes/ capillary endothelial cells may contribute, at least in part, to the repair and regeneration of fibrotic liver through the caveolin-1 signal in the advanced stage of NASH.

\section{Acknowledgments}

The authors thank Hitoshi Yamazaki and Yoshihito Takahashi of Kitasato University Medical Center, and
Takuji Noro, Nobuhiro Tsutsui, Eigorou Yamanouchi, and Hirotaka Tanabe of International University of Health and Welfare Hospital for providing liver specimens. The authors also acknowledge Hajime Imahana of Kitasato University Medical Center for statistical analysis, and Mariko Ogi and Tomoko Yoshii for technical assistance.

\section{Conflicts of interest}

Hiroaki Yokomori received the funding supported by Pfizer Inc., Bristol-Myers Squibb, Merck Sharp \& Dohme Corp., AbbVie GK, Astellas Pharma Inc., and Astrazeneca International PLC, but none primarily designed or interpreted the study.

\section{Abbreviations}

aTGF- $\beta=$ active form of transforming growth factor- $\beta$; CK-19 = cytokeratin 19; ECM = extracellular matrix; HCC = hepatocellular carcinoma; HPCs = hepatic progenitor cells; HSCs = hepatic stellate cells; LSECs = liver sinusoidal endothelial cells; IEM = immunoelectron microscopy; IHC $=$ immunohistochemistry; $\mathrm{KCs}=$ Kupffer cells; ITGF- $\beta=$ latent form of transforming growth factor- $\beta$; MMP-1 = matrix metalloproteinase-1; NAFLD = nonalcoholic fatty liver disease; $\mathrm{NASH}=$ nonalcoholic steatohepatitis; TLR = toll-like receptor

\section{Supplementary data}

Supplementary data associated with this article can be found, at http://nobleresearch.org/Doi/10.14312/23976845.2016-3.ThesedataincludeAppendix-I(Supplementary materials and methods) and Appendix-II (Supplementary Table and Figures).

\section{References}

[1] Puri P, Sanyal AJ. Nonalcoholic fatty liver disease. In: Zakim and Boyer's Hepatology, Sixth edition. Edited by Boyer TD, Manns MP, Sanyal A J. Philadelphia, PA., Saunders, 2006, pp. 941-968.

[2] Pinzani M. Pathophysiology of non-alcoholic steatohepatitis and basis for treatment. Dig Dis. 2011; 29(2):243-248.

[3] Bhala N, Jouness RI, Bugianenesi E. Epidemiology and natural history of patients with NAFLD. Curr Pharm Des. 2013; 19(29):5169-5176.

[4] Matteoni CA, Younossi ZM, Gramlich T, Boparai N, Liu YC, et al. Nonalcoholic fatty liver disease: a spectrum of clinical and pathological severity. Gastroenterology. 1999; 116:1413-1419.

[5] Hashimoto E, Tokushige K. Prevalence, gender, ethnic variations, and prognosis of NASH. J Gastroenterol. 2011; 46 Suppl 1:63-69.

[6] Okazaki I, Noro T, Tsutsui N, Yamanouchi E, Kuroda $H$, et al. Fibrogenesis and carcinogenesis in nonalcoholic steatohepatitis (NASH): Involvement of matrix metalloproteinases (MMPs) and tissue inhibitors of metalloproteinase (TIMPs). Cancers. 2014; 6(3):12201255.

[7] Seki E, Brenner DA. Toll-like receptors and adaptor molecules in liver disease: update. Hepatology. 2008; 48(1):322-335.

[8] Henao-Mejia J, Elinav E, Jin C, Hao L, Mehal WZ, et al. Inflammasome mediated dysbiosis regulates progression of NAFLD and obesity. Nature. 2012; 482(7384):179-185.

[9] Friedman SL. Liver fibrosis in 2012: Convergent pathways that cause hepatic fibrosis in NASH. Nat Rev Gastroenterol Hepatol. 2013; 10(2):71-72.

[10] Boulter L, Govaere O, Bird TG, Radulescu S, Ramachandran P, et al. Macrophage derived Wnt opposes Notch signaling to specify hepatic progenitor cell fate in chronic liver disease. Nat Med. 2012; 18(4):572579. 
[11] Diehl AM. Neighborhood watch orchestrates liver regeneration. Nat Med. 2012; 18(4):497-499.

[12] Yang L, Roh YS, Song J, Zhang B, Liu C, et al. Transforming growth factor beta signaling in hepatocytes participates in steatohepatitis through regulation of cell death and lipid metabolism in mice. Hepatology. 2014; 59(2):483-495.

[13] Okazaki I, Nabeshima K. Introduction: MMPs, ADAMs/ADAMTSs research products to achieve big dream. Anticancer Agents Med Chem. 2012; 12(7):688-706.

[14] limuro Y, Nishio T, Morimoto T, Nitta T, Stefanovic B, et al. Delivery of matrix metalloproteinase-1 attenuates established liver fibrosis in the rat. Gastroenterology. 2003; 124(2):445-458.

[15] Saunders WB, Bayless KJ, Davis GE. MMP-1 activation by serine proteases and MMP-10 induces human capillary tubular network collapse and regression in 3D collagen matrices. J Cell Sci. 2005; 118 (Pt 10):2325-2340.

[16] Kunisch E, Kinne RW, Alsalameh RJ, Alsalameh S. Pro-inflammatory IL-beta and/or TNF-alpha up-regulate matrix metalloproteases-1 and -3 mRNA in chondrocyte subpopulations potentially pathogenic in osteoarthritis: in situ hybridization studies on a single cell level. Int J Rheum Dis. 2014.

[17] Brunt EM, Janney CG, Di Bisceglie AM, Neuschwander-Tetri BA, Bacon BR. Nonalcoholic steatohepatitis: a proposal for grading and staging the histological lesions. Am J Gastroenterol. 1999; 94(9):2467-2474.

[18] Kleiner DE, Brunt EM, Van Natta M, Behling C, Contos MJ, et al. Nonalcoholic Steatohepatitis Clinical Research Network: Design and validation of a histological scoring system for nonalcoholic fatty liver disease. Hepatology. 2005; 41(6):1313-1321.

[19] Hara M, Kirita A, Kondo W, Matsuura T, Nagatsuma K, et al. LAP degradation product reflects plasma kallikrein-dependent TGFactivation in patients with hepatic fibrosis. SpringerPlus. 2014; 3:221.

[20] Yamazaki H, Oda M, Takahashi Y, Iguchi H, Yoshimura K, et al. Relation between ultrastructural localization, changes of caveolin-1 and capillarization of liver sinusoidal endothelial cells in human hepatitis c-related cirrhotic liver. J Histochem Cytochem. 2013; 61(2):169-176.

[21] Qiu Y, Liu S, Chen HT, Yu CH, Teng XD, et al. Upregulation of caveolin-1 and SR-B1 in mice with non-alcoholic fatty liver disease. Hepatobiliary Pancreat Dis Int. 2013, 12(6):630-636.

[22] Frank PG, Woodman SE, Park DS, Lisanti MP. Caveolin, caveolae, and endothelial cell function. Arterioscler Thromb Vasc Biol. 2003; 23(7):1161-1168.

[23] Felicetti F, Parolini I, Bottero L, Fecchi K, Errico MC, et al. Caveolin-1 tumor-promoting role in human melanoma. Int J Cancer. 2009; 125(7):1514-1522.

[24] Annabi B, Lachambre M, Bousquet-Gagnon N, Pagé M, Gingras D, et al. Localization of membrane-type 1 matrix metalloproteinase in caveolae membrane domains. Biochem J. 2001; 353(Pt 3):547-553.

[25] Tang W, Hemler ME. Caveolin-1 regulates MMP-1 induction and CD147/ EMMPRIN cell surface clustering. J Biol Chem. 2004; 279(12):1111211118.

[26] Tang W, Chang SB, Helmer ME. Links between CD147 function, glycosylation, and caveolin-1. Mol Biol Cell. 2004; 15(9):4043-4050.

[27] Sica A, Invernizzi P, Mantovani A. Macrophage plasticity and polarization in liver homeostasis and pathology. Hepatology. 2014; 59(5):20352043.

[28] Pollard JW. Tropic macrophages in development and disease. Nat Rev Immunol. 2009; 9(4):259-270.

[29] Baker N, Tuan RS. The less-often-traveled surface of stem cells: caveolin-1 and caveolae in stem cells, tissue repair and regeneration. Stem Cell Res Ther. 2013; 4(4):90.

[30] Ding BS, Cao Z, Lis R, Nolan DJ, Guo P, et al. Divergent angiocrine signals from vascular niche balance liver regeneration and fibrosis. Nature. 2014; 505(7481):97-102.

[31] Ding BS, Nolan DJ, Butler JM, James D, Babazadeh AO, et al. Inductive angiocrine signals from sinusoidal endothelium are required for liver regeneration. Nature. 2010; 468(7321):310-315.

[32] Hu J, Srivastava K, Wieland M, Runge A, Mogler C, et al. Endothelial cellderived angiopoietin-2 controls liver regeneration as a spatiotemporal rheostat. Science. 2014; 343(6169):416-419.

[33] Shah V, Haddad FG, Garcia-Cardena G, Frangos JA, Mennone A, et al. Liver sinusoidal endothelial cells are responsible for nitric oxide modulation of resistance in the hepatic sinusoids. J Clin Invest. 1997; 100(11):2923-2930.

[34] Yokomori H, Oda M, Yoshimura K, Nomura M, Wakabayashi G, et al. Elevated expression of caveolin-1 at protein and mRNA level in human cirrhotic liver: relation with nitric oxide. J Gastroenterol. 2003; 38(9):854-860 